\title{
Intracranial Atherosclerotic Plaque Enhancement in Patients with Ischemic Stroke
}

\author{
M. Skarpathiotakis, D.M. Mandell, R.H. Swartz, G. Tomlinson, and D.J. Mikulis
}

\begin{abstract}
BACKGROUND AND PURPOSE: Inflammation of an atherosclerotic plaque is a well-known risk factor in the development of ischemic stroke and myocardial infarction. MR imaging is capable of characterizing inflammation by assessing plaque enhancement in both extracranial carotid arteries and coronary arteries. Our goal was to determine whether enhancing intracranial atherosclerotic plaque was present in the vessel supplying the territory of infarction by using high-resolution vessel wall MR imaging.
\end{abstract}

MATERIALS AND METHODS: High-resolution vessel wall 3T MR imaging studies performed in 29 patients with ischemic stroke and intracranial vascular stenoses were reviewed for presence and strength of plaque enhancement.

RESULTS: Sixteen patients were studied during the acute phase ( $<4$ weeks from acute stroke), 5 patients in the subacute phase $(4-12$ weeks), and 8 patients in the chronic phase ( $>12$ weeks) of the ischemic injury. In all of the acute phase patients, atherosclerotic plaque in the vessel supplying the stroke territory demonstrated strong enhancement. There was a trend of decreasing enhancement as the time of imaging relative to the ischemic event increased.

CONCLUSIONS: Strong pathologic enhancement of intracranial atherosclerotic plaque was seen in all patients imaged within 4 weeks of ischemic stroke in the vessel supplying the stroke territory. The strength and presence of enhancement of the atherosclerotic plaque decreased with increasing time after the ischemic event. These findings suggest a relationship between enhancing intracranial atherosclerotic plaque and acute ischemic stroke.

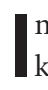
nflammation related to an atherosclerotic plaque is a wellknown risk factor in the development of ischemic stroke related to extracranial carotid plaques and myocardial infarction related to coronary artery plaques. ${ }^{1-3} \mathrm{MR}$ imaging characterization of extracranial atherosclerotic plaque has been well studied and well established. ${ }^{4-6}$ Extracranial artery inflammatory plaques have been reported to show asymmetric wall thickening with enhancement on MR imaging. ${ }^{7}$

High-resolution vessel wall $3 \mathrm{~T}$ MR imaging is a fairly new technique that has allowed a more detailed examination of smallsized and deeply located intracranial arteries. Although early work using 3T MR imaging to characterize intracranial atherosclerotic plaques showed asymmetric wall thickening, ${ }^{8}$ no work has yet

Received January 16, 2012; accepted after revision May 14.

From the Department of Medical Imaging, Toronto Western Hospital, Toronto, Ontario, Canada.

Previously presented as an electronic poster at: Annual Meeting of the American Society of Neuroradiology, April 21-26, 2012; New York, New York.

Please address correspondence to Dr. David Mikulis, Toronto Western Hospital, Department of Medical Imaging, Rm 3MC-431, 399 Bathurst St, Toronto, Ontario, M5T Canada; e-mail: 2S8mikulis@mac.com

http://dx.doi.org/10.3174/ajnr.A3209 been performed regarding the enhancement of intracranial atherosclerotic plaque as an indicator of inflammation or association with ischemic stroke. The goal of this research was to assess the correlation between the presence and timing of enhancing intracranial atherosclerotic plaques and the territory of ischemic stroke in patients who have undergone specialized vessel wall 3T MR imaging at a tertiary stroke center.

Our hypothesis was based on our understanding of the increased risk of ischemic stroke or myocardial infarction secondary to cervical and coronary atherosclerotic plaque inflammation, and on MR imaging being capable of detecting extracranial plaque inflammation in the form of contrast enhancement. Therefore, we planned to test 2 hypotheses in patients with intracranial atherosclerotic plaques presenting with acute intracranial infarction: 1 ) only plaques in the vessel that supplies the territory of infarction will show enhancement; and 2) plaque enhancement decreases or resolves over time after the acute stroke event.

\section{MATERIALS AND METHODS \\ Subjects}

This study was a retrospective analysis of consecutive patients who have undergone vessel wall 3T MR imaging at a tertiary care 
Table 1: Sequence parameters for vessel wall imaging protocol

\begin{tabular}{lcccccccc}
\hline & $\begin{array}{c}\text { TR } \\
\text { (msec) }\end{array}$ & $\begin{array}{c}\text { TE } \\
\text { (msec) }\end{array}$ & $\begin{array}{c}\text { TI } \\
\text { (msec) }\end{array}$ & $\begin{array}{c}\text { Echo-Train } \\
\text { Length }\end{array}$ & Section Thickness & $\begin{array}{c}\text { Parallel } \\
\text { Factor }\end{array}$ & FOV & Resolution \\
\hline $\begin{array}{c}\text { T1 FLAIR pre- and postgadolinium } \\
\text { (axial and coronal or sagittal) }\end{array}$ & 2108 & 12 & 860 & 6 & $\begin{array}{r}2 \mathrm{~mm} \text { (initially } 3 \mathrm{~mm}, \\
\text { changed to } 2 \mathrm{~mm})\end{array}$ & 2 & $16 \times 22 \mathrm{~cm}$ & $384 \times 384$ \\
$\begin{array}{l}\text { T2 FRFSE (axial and coronal) } \\
\text { 2 }\end{array}$ & 3450 & 92 & - & 17 & $\begin{array}{r}\text { mm (initially } 3 \mathrm{~mm}, \\
\text { changed to } 2 \mathrm{~mm} \text { ) }\end{array}$ & 2 & $16 \times 22 \mathrm{~cm}$ & $512 \times 512$ \\
\hline
\end{tabular}

Note:-FRFSE indicates fast-recovery fast spin-echo.

Table 2: Imaging performed in the acute phase

\begin{tabular}{cc}
$\begin{array}{c}\text { Plaques in a Single } \\
\text { Vascular Territory } \\
\text { in } 13 \text { Patients }\end{array}$ & $\begin{array}{c}\text { Plaques in Multiple } \\
\text { Vascular Territories } \\
\text { in } 3 \text { Patients }\end{array}$ \\
\hline $\begin{array}{c}\text { Solitary plaque in } 10 / 13 \\
\text { All plaques enhanced }\end{array}$ & Plaque in stroke territory \\
Multiple plaques in 3/13 & Solitary plaque in $3 / 3$ \\
All plaques enhanced & All enhanced \\
& Plaque in nonstroke territory \\
& Solitary plaque in $2 / 3$ \\
& Multiple plaque enhanced \\
& All plaques enhanced \\
\hline
\end{tabular}

center (Toronto Western Hospital). A search of the institution's PACS revealed a total of 126 specialized 3T vessel wall imaging studies performed from February 2006 to October 2010. Patients were originally selected to undergo the specialized vessel wall imaging technique because luminal narrowing was demonstrated on MRA, and, therefore, the patients in our study were a subset of a larger group with vascular stenoses of any etiology. Patients were selected for inclusion if they had documented prior ischemic stroke (based on diffusion-weighted imaging) and evidence of at least 1 intracranial atherosclerotic plaque (based on MRA and vessel wall imaging). Patients with ischemic stroke attributable to other causes, such as vasculitis or absence of intracranial atherosclerotic plaque, were excluded. In addition, patients with extracranial atherosclerotic plaque and no intracranial atherosclerotic plaque were excluded.

Twenty-nine of 126 vessel wall MR imaging studies met these criteria. The following patient information was collected: patient age, sex, date of study, date of stroke presentation, location of ischemic stroke, site of atherosclerotic plaque(s), presence and strength (strong or mild) of enhancement of atherosclerotic plaque(s), and the results of other studies, if performed, including MRA or CTA of the neck vessels, conventional angiography, and echocardiogram. The age range of the patients included in the study was 35-84 years, with an average of 61.2 years. Eleven of the imaging studies were performed in female patients.

The MR images were acquired using vendor-supplied sequences approved for clinical use. Hospital research ethics board approval was obtained for a systematic, retrospective chart and imaging review.

\section{Imaging Protocol}

Patients were scanned on a 3T MR imaging system (HDX platform; GE Healthcare, Milwaukee, Wisconsin) using an 8-channel head coil. All sequences applied were standard, approved, vendorsupplied pulse sequences. The "wall imaging protocol" used in this study implemented a standardized set of sequences with targeted axial, coronal, and sagittal sampling of the site of intracra- nial stenosis (imaging of the entire brain was not performed). All sequences consisted of thin 2D slabs of 2-mm thickness through the area of interest. The neuroradiologist selected the target vessels, which were those that had stenoses and supplied the territory of stroke, and chose the best combination of acquisition orientations (axial and coronal, or axial and sagittal) according to the prior MRA findings. Typically, either the anterior or posterior circulation was imaged to the level of the circle of Willis depending on the location of the target lesion. All sequences were monitored for quality to ensure that the affected artery at the site of stenosis was well visualized in 2 planes. At least 1 of the 2 planes gave an optimal section through the target vessel. Axial and coronal T2 fast-recovery fast spin-echo sequences were obtained. These were followed by pre- and postgadolinium (7.5-mL Gadovist; Bayer Schering Pharma, Berlin, Germany) axial and coronal or sagittal T1 FLAIR sequences, with or without fat saturation (Table 1). The postgadolinium images were obtained immediately after injection for all patients.

\section{Analysis}

An experienced senior neuroradiologist assessed the studies. Although aware of the study objectives, the reader was blinded to time relative to the date of stroke, the diffusion details, and the MRA findings. The reader was provided with the vessel wall imaging sequences (T2, pre-, and postgadolinium T1 FLAIR) and analyzed these for the presence or absence of plaque enhancement and strength of enhancement classified as mild or strong. The T2 and noncontrast T1 FLAIR images were used to distinguish the vessel wall from the lumen via its black-blood characteristics, absence of blood signal, and decreased sensitivity to flow artifacts. Flow artifacts, including slow flow, did not pose ambiguity of findings in any of the cases. The T2 and noncontrast T1 FLAIR sequences were also used to delineate arterial walls and distinguish the arterial findings from adjacent venous structures.

The postgadolinium T1 FLAIR images were compared with the pregadolinium T1 FLAIR images to determine presence or absence and strength of enhancement. A subjective analysis was performed, where enhancement was compared with the pituitary parenchyma. If plaque enhancement was similar to pituitary enhancement, it was deemed strong; if less than the pituitary, it was deemed mild; and if there was no change compared with the noncontrast images, it was deemed absent.

Imaging was classified as acute phase if it was performed within 4 weeks of the presenting symptoms, subacute phase if between 4-12 weeks from the presenting symptoms, and chronic phase if performed beyond 12 weeks from presentation.

The acute phase cases were divided into 2 groups: those with an atherosclerotic plaque in a single vascular territory (the territory of stroke) and those with plaques in multiple vascular terri- 

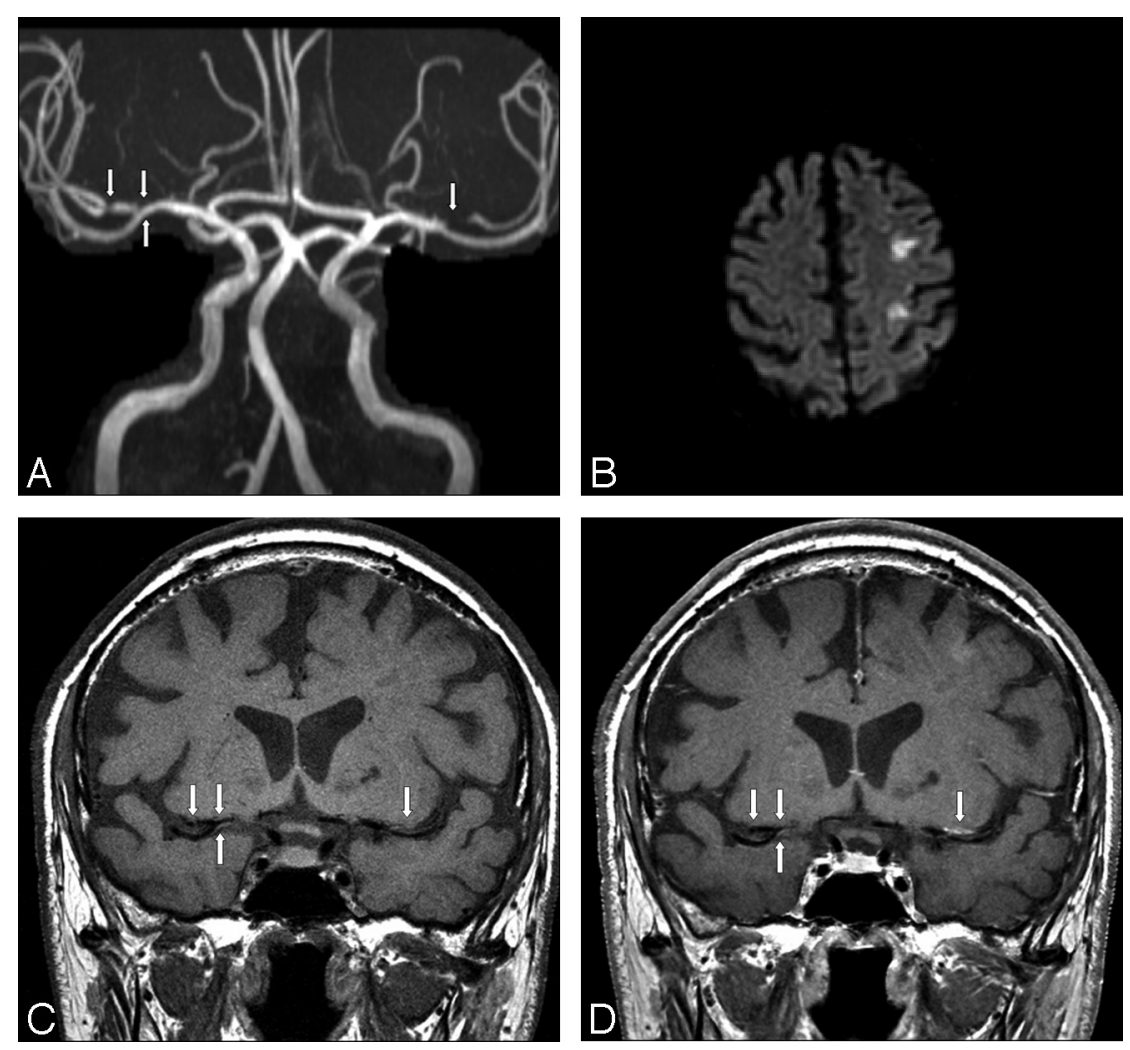

FIG 1. A, 3D reformatted MRA image demonstrating bilateral MCA stenoses (arrows). B, Diffusion-weighted image of the brain demonstrating areas of restricted diffusion/infarction in the left MCA territory. C, Coronal noncontrast T1-weighted image through the level of the MCAs. Atherosclerotic plaque is present in both the right MCA and left MCA (arrows) at the site of stenoses seen in $A$. D, Coronal postcontrast T1-weighted image at the same level as in $C$, with enhancement of the left MCA atherosclerotic plaque but not of the plaques on the right.

tories (the territory of stroke and other territories). Of the plaques in the vascular territory of the stroke, these were categorized into a solitary plaque versus multiple or tandem plaques. The presence of enhancement in these plaques was tabulated. Similarly, in cases with plaque in multiple vascular territories, the analysis for solitary versus multiple or tandem plaques and enhancement was tabulated for each of the territories.

The level of enhancement of an atherosclerotic plaque in a vessel supplying the stroke territory was determined qualitatively as absent, mild, or strong. Comparison of enhancement of atherosclerotic plaque in acute, subacute, and chronic stroke was performed by tabulating the number of cases demonstrating absent, mild, or strong enhancement for each of the timeframes for plaques in the vessels supplying the stroke territory. The Fisher exact test was used to test for an association between time and level of enhancement, and the Spearman correlation, along with its $95 \%$ confidence intervals, was used to quantify the strength of association. The level of enhancement was also plotted relative to time from the initial stroke presentation.

Six patients had wall imaging performed a second time after the ischemic event because repeat vessel wall imaging was requested by the referring service on clinical follow-up. These patients did not have repeat ischemic events. A separate analysis was performed for these 6 patients to look at the change in plaque enhancement over time. Statistical graphs and analyses were completed using R version 2.14 (http://www.r-project.org/), and a $P$ value less than .05 was taken to indicate statistical significance. ${ }^{9}$

\section{RESULTS}

Imaging was performed during the acute phase of the stroke in 16 cases (Table 2). Of these, 13 patients had 1 or multiple plaques in vessels supplying only the territory of the stroke, with all other vascular territories plaque free. Of these, 10 patients had solitary plaque and 3 had multiple intracranial plaques. All plaques demonstrated strong enhancement. There were 3 patients in the acute stroke phase who had plaques in both the stroke territory and nonstroke territory vasculature. The plaques in the stroke territory were solitary in all 3 cases and all
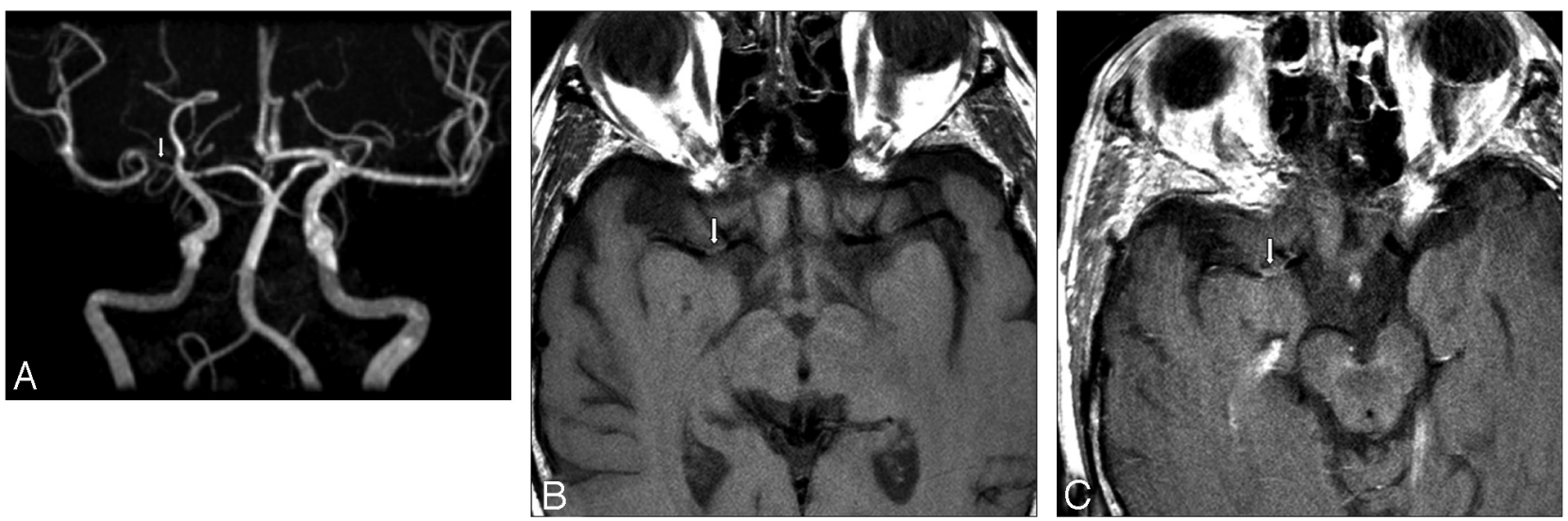

FIG 2. A, 3D reformatted MRA image demonstrating right MCA stenoses (arrow). B, Axial noncontrast T1-weighted image through the level of the MCAs. Atherosclerotic plaque is present in the right MCA (arrow) at the site of stenosis seen in A. C, Axial postcontrast T1-weighted image at the same level as in $B$, with enhancement of the right MCA atherosclerotic plaque. 

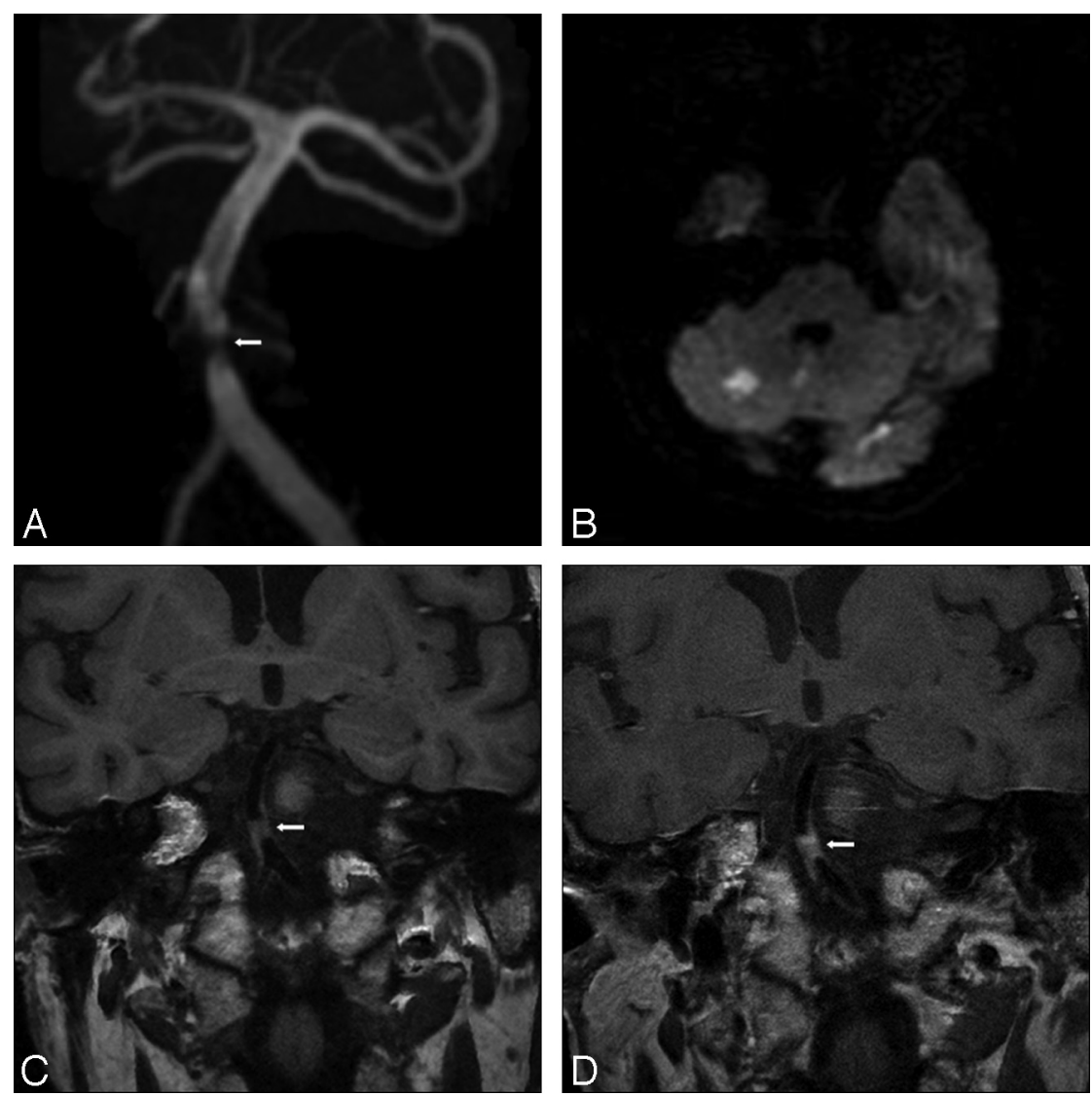

FIG 3. $A, 3 D$ reformatted MRA image demonstrating midbasilar artery stenosis (arrow). B, Diffusion-weighted image of the brain demonstrating areas of restricted diffusion/infarction in the basilar artery territory. C, Coronal noncontrast T1-weighted image through the level of the basilar artery. Atherosclerotic plaque is present (arrow) at the site of stenosis seen in A. D, Coronal postcontrast T1-weighted image at the same level as in $C$, with enhancement of midbasilar atherosclerotic plaque.

demonstrated strong enhancement. One of the 3 patients had a solitary plaque in a nonstroke territory vessel that did not enhance, another had a solitary plaque in a nonstroke territory vessel that showed mild enhancement, and the third had multiple plaques in nonstroke territory vessels that showed strong enhancement. Extracranial artery MR or CT angiography results were available for all of the 16 patients imaged in the acute phase of stroke. None showed significant atherosclerotic disease ( $>70 \%$ stenosis) in the extracranial feeding vessel to the intracranial vessels of the stroke territory. Figure 1 shows the MRA, diffusion-

Table 3: Time since infarct by enhancement

\begin{tabular}{lcccc}
\hline & \multicolumn{4}{c}{ Enhancement } \\
\cline { 2 - 5 } Time Since Infarct & None & Mild & Strong & Total \\
\hline Acute phase (0-4 weeks) & 0 & 0 & 16 & 16 \\
& $(0 \%)$ & $(0 \%)$ & $(100 \%)$ & $(55.2 \%)$ \\
Subacute phase (4-12 weeks) & 1 & 3 & 1 & 5 \\
& $(20 \%)$ & $(60 \%)$ & $(20 \%)$ & $(17.2 \%)$ \\
Chronic phase ( $>12$ weeks) & 4 & 3 & 1 & 8 \\
& $(50 \%)$ & $(37.5 \%)$ & $(12.5 \%)$ & $(27.6 \%)$ \\
Total & 5 & 6 & 18 & 29 \\
& $(17.2 \%)$ & $(20.7 \%)$ & $(62.1 \%)$ & $(100 \%)$ \\
\hline
\end{tabular}

Note:-Fisher exact test for count data, $P$ value $=2.8 \times 10^{-6}$. Spearman correlation $=-0.84(95 \% \mathrm{Cls}-0.92,-0.70)$. weighted, and pre- and postgadolinium T1 FLAIR images of a patient in the acute phase of infarction, with bilateral MCA atherosclerotic plaque. Postgadolinium enhancement is present only on the side of infarction. Figures 2 and 3 illustrate 2 other examples of stenoses in patients with stroke with atherosclerotic plaque that enhances in the right MCA and basilar arteries, respectively.

There were 5 subacute phase strokes with atherosclerotic plaque in the intracranial vessel supplying the territory of stroke. One showed strong enhancement, 3 showed mild enhancement, and 1 showed no enhancement (Table 2). There were no patients in the subacute category who had intracranial plaques in a nonstroke territory vessel. Extracranial artery MR angiography results were available only for the 1 case showing strong enhancement of the intracranial vessel supplying the stroke territory. There was no abnormality of the feeding vessel to the intracranial vessel supplying the stroke territory.

There were 8 chronic phase strokes with atherosclerotic plaque(s) in the vessel(s) supplying the territory of stroke. One showed strong enhancement, 3 showed mild enhancement, and 4 showed no enhancement (Table 3 ). The 1 patient showing strong enhancement, and 1 of the 3 showing mild enhancement, were imaged in what we defined as the chronic phase but were only 17 and 18 weeks from the date of stroke presentation, respectively. The other 2 patients showing mild enhancement in the chronic phase were imaged at 6.5 months and 1.5 years after stroke presentation. The remaining 4 cases not showing enhancement ranged from 18.5 weeks to 3 years after stroke presentation. With regard to nonstroke territory vessel plaques, 2 of the chronic phase cases had plaque, with 1 showing mild enhancement and the other no enhancement. Extracranial artery MR or CT angiography results were available for 2 of these chronic phase cases, 1 with mild and the other with strong enhancement of the intracranial vessel supplying the stroke territory. Neither of the patients showed an abnormality of the feeding vessel to the intracranial vessels supplying the stroke territory.

When considering strength and presence of enhancement in the atherosclerotic plaques versus time from initial stroke presentation (Fig 4), all the plaques enhanced near the time of stroke. There is a trend toward decreased or absent plaque enhancement with increased time after stroke presentation (Spearman correlation $=-0.84 ; 95 \%$ CIs $-0.92,-0.70)$, which is also significant according to the Fisher exact test $\left(P\right.$ value $\left.=2.8 \times 10^{-6}\right)$. In 6 patients with wall imaging performed twice, enhancement either persisted over time or decreased (Fig 5). 


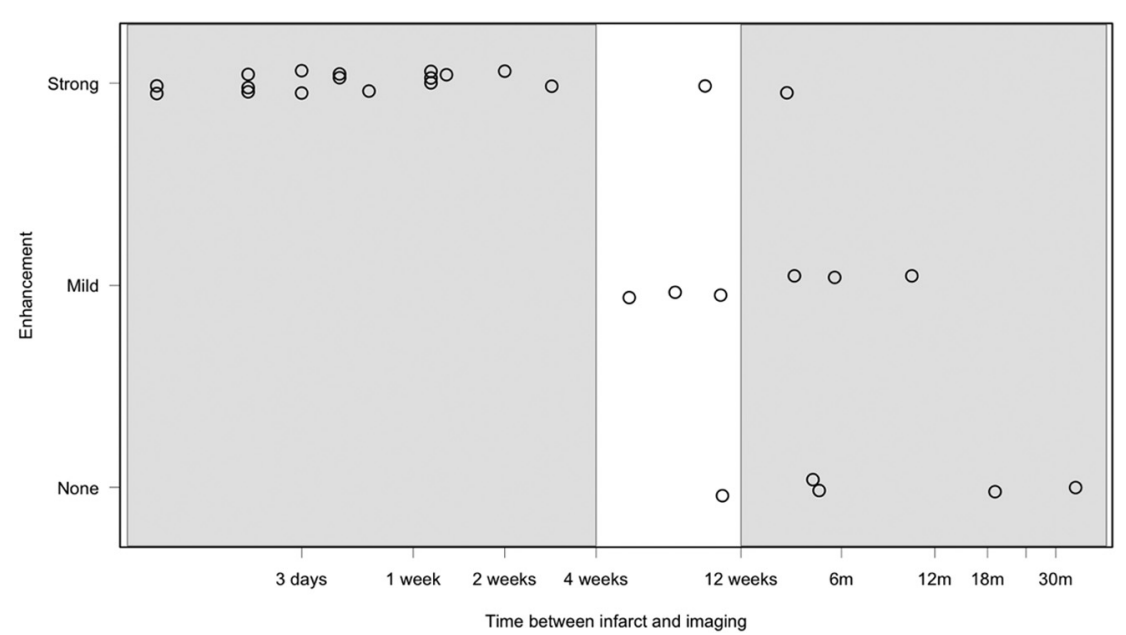

FIG 4. Strength and presence of enhancement versus time elapsed between imaging and initial stroke presentation on a logarithmic scale. Acute, subacute, and chronic timeframes are highlighted.

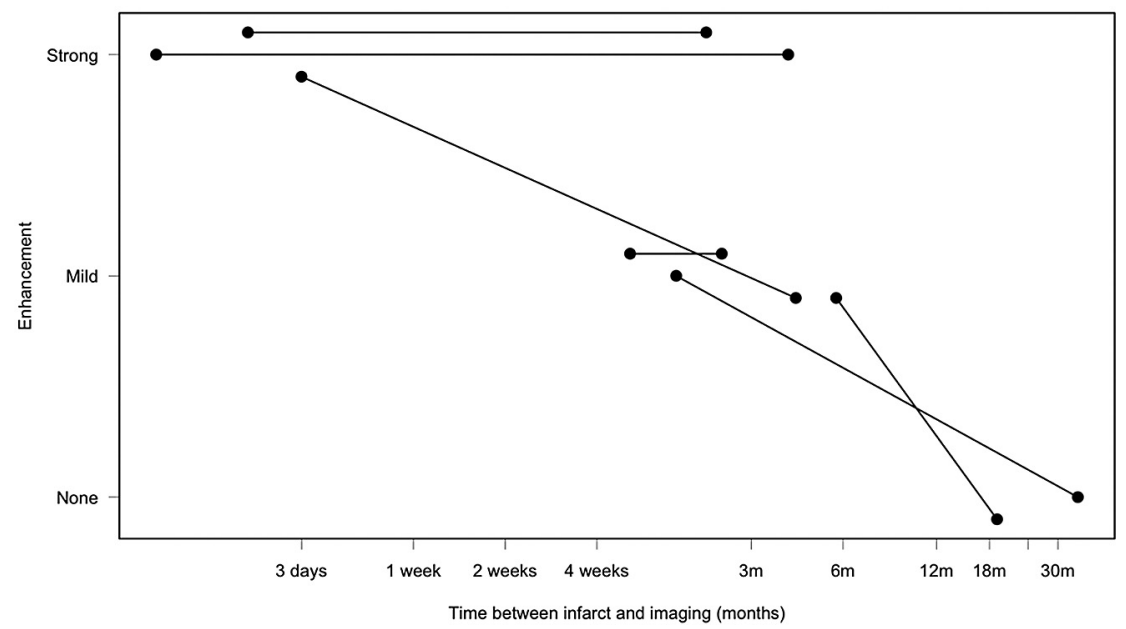

FIG 5. Strength and presence of enhancement versus time elapsed between imaging and initial stroke presentation for 6 patients in whom imaging was performed at 2 separate time points.

\section{DISCUSSION}

The results of this study showed strong enhancement of intracranial atherosclerotic plaque in the vessel supplying the stroke territory in all 16 patients who were imaged within 4 weeks of ischemic stroke. In patients imaged after more than 4 weeks from the time of stroke, enhancement of the plaque in the vessel supplying the territory of stroke was mild or absent in 11 of 13 cases.

This work is novel in that it addresses the physiology of intracranial atherosclerotic plaque and ischemic stroke. The study raises the question as to whether gadolinium enhancement is a marker of intracranial plaque inflammation, and whether intracranial plaque inflammation and intracranial plaque rupture are risk factors for acute ischemic stroke. We are more familiar with plaque inflammation, plaque enhancement, and plaque rupture in the extracranial carotid and coronary arteries, but there is limited information regarding intracranial vessels.

Regarding extracranial plaques, inflammatory mechanisms play a central role in the pathogenesis and progression of extracranial atherosclerotic plaque, plaque rupture, thrombosis, and stroke. ${ }^{1}$ Previous studies have shown a strong relationship between serum inflammatory markers-most reliably, C-reactive protein - and the risk of future stroke or myocardial infarction. ${ }^{2}$ In addition to serum markers, the use of noninvasive imaging modalities including $\mathrm{CT}$, MR imaging, and nuclear medicine for the characterization of plaque inflammation has been gaining interest over the past several years., ${ }^{30-17}$ Investigations with FDG-PET imaging have shown that it may be used as a surrogate marker for inflammation of plaques, as patients with the highest levels of FDG uptake had the greatest concentrations of inflammatory biomarkers. ${ }^{10,11}$

MR imaging characterization of extracranial atherosclerotic plaque has been well studied and well established. ${ }^{4-6}$ Several aspects of plaque structure, including size, wall thickness, integrity of the fibrous cap, and the presence of inflammation, can be assessed using MR imaging. ${ }^{13,18-21} \mathrm{MR}$ imaging characteristics of the aorta and carotid arteries, including enhancement, have been shown to be associated with elevated serum markers of inflammation. ${ }^{3,14}$ One study using cardiac MR imaging in patients with acute myocardial infarction demonstrated enhancement of the coronary vessel wall present during the acute phase, with a decrease in enhancement after 3 months in the chronic phase in parallel with levels of C-reactive protein. ${ }^{12}$

MR imaging studies of the extracranial carotid arteries have identified features of an atherosclerotic plaque that may convey increased risk of thromboembolic events, even in cases with less than $50 \%$ luminal stenosis. ${ }^{5}$ Extracranial large artery inflammatory plaques have been reported to demonstrate asymmetric wall thickening with gadolinium enhancement. ${ }^{7}$

Given previous findings related to extracranial plaque, one might postulate that similar physiology exits in the intracranial vessels regarding risk factors for ischemic stroke. The findings of this study regarding strong enhancement of intracranial plaques within 4 weeks of ischemic stroke are similar to the findings for extracranial plaques and may therefore suggest that intracranial enhancement is a marker of inflammation. Inflammation and, therefore, plaque enhancement are expected to decrease in the chronic phase for extracranial vessels, ${ }^{3,12}$ and this was also seen in this study.

An important limitation of this exploratory cross-sectional study was that the number of cases is small. A larger number of cases with longitudinal prospective studies is needed for validation in order to determine whether atherosclerotic plaque enhancement precedes or is in close relationship to an ischemic 
stroke. Another limitation of the study is that information on the carotid arteries was not available in 10 of 13 patients in the subacute and chronic imaging phases; therefore, we could not exclude an extracranial source of stroke in these patients. An additional limitation is that when performing the vessel wall imaging sequences on the target vessels, either the anterior circulation or the posterior circulation was imaged to the level of the circle of Willis. Nonstroke territory plaques were identified if they were in the portion of the brain imaged, and it is possible that some nonculprit atherosclerotic plaques may have been missed because of the restricted coverage. Finally, our study was limited in its inability to determine the false-positives and false-negatives because pathologic evidence on the intracranial plaques was not obtained.

\section{CONCLUSIONS}

MR intracranial vessel imaging of patients with intracranial atherosclerosis showed plaque enhancement in the vessel supplying the territory of stroke in all patients studied within 4 weeks of the onset of ischemia. There is a trend toward a decrease or resolution of enhancement with time relative to the initial stroke presentation, similar to findings seen in MR imaging evaluation of extracranial carotid arteries and coronary arteries. The findings suggest at least a relationship between intracranial atherosclerotic plaque enhancement and acute ischemic stroke. If these results are analogous to the findings seen in extracranial vessels, intracranial plaque enhancement could be a marker of plaque inflammation.

Disclosures: Richard Swartz-UNRELATED: Grants/Grants Pending: Roche;* Payment for Lectures (including service on speakers bureaus): Bristol-Myers Squibb. David Mikulis_UNRELATED: Grants/Grants Pending: Ontario Research Fund;* Payment for Lectures (including service on speakers bureaus): International Diagnostic Course Davos; Patents (planned, pending, or issued): Patent pending for rebreathing device - RespirAct; Payment for Development of Educational Presentations: International Diagnostic Course Davos; Stock/Stock Options: Equity position in Thornhill Research, Comments: RespirAct vendor. (*Money paid to institution)

\section{REFERENCES}

1. Elkind MSV. Inflammatory mechanisms of stroke. Stroke 2010;41:S3-8

2. Willerson JT, Ridker PM. Inflammation as a cardiovascular risk factor. Circulation 2004;109:II2-10

3. Wasserman BA. Advanced contrast-enhanced MRI for looking beyond the lumen to predict stroke: building a risk profile for carotid plaque. Stroke 2010;41:S12-16

4. Yuan C, Mitsumori LM, Beach KW, et al. Carotid atherosclerotic plaque: noninvasive MR characterization and identification of vulnerable lesions. Radiology 2001;221:285-99

5. Wasserman BA, Wityk RJ, Trout HH III, et al. Low-grade carotid stenosis: looking beyond the lumen with MRI. Stroke 2005;36: 2504-13

6. Koops A, Ittrich H, Petri S, et al. Multicontrast-weighted magnetic resonance imaging of atherosclerotic plaques at 3.0 and 1.5 Tesla: ex-vivo comparison with histopathologic correlation. Eur Radiol 2007; 17:279-86

7. Adams GJ, Greene J, Vick GW III, et al. Tracking regression and progression of atherosclerosis in human carotid arteries using high-resolution magnetic resonance imaging. Magn Reson Imaging 2004;22:1249-58

8. Swartz RH, Bhuta SS, Farb RI, et al. Intracranial arterial wall imaging using high-resolution 3-Tesla contrast-enhanced MRI. Neurology 2009;72:627-34

9. R Development Core Team (2012). R: A Language and Environment for Statistical Computing. R Foundation for Statistical Computing, Vienna, Austria. http://www.R-project.org/

10. Rudd JH, Narula J, Strauss HW, et al. Imaging atherosclerotic plaque inflammation by fluorodeoxyglucose with positron emission tomography: ready for prime time? J Am Coll Cardiol 2010;55: 2527-35

11. Rudd JH, Myers KS, Bansilal S, et al. Relationships among regional arterial inflammation, calcification, risk factors, and biomarkers: a prospective fluorodeoxyglucose positron-emission tomography/ computed tomography imaging study. Circ Cardiovasc Imaging 2009;2:107-15

12. Ibrahim T, Makowski MR, Jankauskas A, et al. Serial contrast-enhanced cardiac magnetic resonance imaging demonstrates regression of hyperenhancement within the coronary artery wall in patients after acute myocardial infarction. JACC Cardiovasc Imaging 2009;2:580-88

13. Rudd JH, Fayad ZA. Imaging atherosclerotic plaque inflammation. Nat Clin Pract Cardiovasc Med 2008;5:S11-17

14. Weiss CR, Arai AE, Bui MN, et al. Arterial wall MRI characteristics are associated with elevated serum markers of inflammation in humans. J Magn Reson Imaging 2001;14:698-704

15. Lerakis S, Synetos A, Toutouzas K, et al. Imaging of the vulnerable plaque: noninvasive and invasive techniques. Am J Med Sci 2008;336:342-48

16. Hamdan A, Assali A, Fuchs S, et al. Imaging of vulnerable coronary artery plaques. Catheter Cardiovasc Interv 2007;70:65-74

17. Bhatia V, Bhatia $R$, Dhindsa $S$, et al. Imaging of the vulnerable plaque: new modalities. South Med J 2003;96:1142-47

18. Fayad ZA, Fuster V. The human high-risk plaque and its detection by magnetic resonance imaging. Am J Cardiol 2001;88:42E-45E

19. Itskovich V, Samber DD, Mani V, et al. Quantification of human atherosclerotic plaques using spatially enhanced cluster analysis of multicontrast-weighted magnetic resonance images. Magn Reson Med 2004;52:515-23

20. Yuan C, Zhang S, Polissar NL, et al. Identification of fibrous cap rupture with magnetic resonance imaging is highly associated with recent transient ischemic attack or stroke. Circulation 2002;105: 181-85

21. Yuan C, Mitsumori LM, Ferguson MS, et al. In vivo accuracy of multispectral magnetic resonance imaging for identifying lipid-rich necrotic cores and intraplaque hemorrhage in advanced human carotid plaques. Circulation 2001;104:2051-56 